\title{
HEAT CONTENT ASYMPTOTICS FOR OPERATORS OF LAPLACE TYPE WITH SPECTRAL BOUNDARY CONDITIONS
}

\author{
P. GILKEY, K. KIRSTEN, AND JH. PARK
}

\begin{abstract}
Let $P$ be an operator of Dirac type and let $D=P^{2}$ be the associated operator of Laplace type. We impose spectral boundary conditions and study the leading heat content coefficients for $D$.
\end{abstract}

\section{INTRODUCTION}

Let $P$ be an operator of Dirac type on a vector bundle $V$ over a compact Riemannian manifold $M$ of dimension $m$ with smooth boundary $\partial M$. Let $D:=P^{2}$ be the associated operator of Laplace type. The leading symbol $\gamma$ of $P$ defines a Clifford module structure on $V$. Choose an auxiliary connection $\nabla$ on $V$ so that $\nabla \gamma=0$. Adopt the Einstein convention and sum over repeated indices; indices $i, j$ will range from 1 to $m$ and index a local orthonormal frame $\left\{e_{i}\right\}$ for $T M$. Expand

$$
P=\gamma_{i} \nabla_{e_{i}}+\psi_{P}
$$

We must impose suitable boundary conditions. As $P$ need not admit local boundary conditions, we shall consider spectral boundary conditions; these were first introduced by Atiyah et. al. [1 to study the index theorem for manifolds with boundary. Near the boundary, normalize the local frame so that $e_{m}$ is the inward unit geodesic vector field. Let indices $a, b$ range from 1 to $m-1$ and index the induced orthonormal frame for $T \partial M$. Let

$$
A=-\gamma_{m} \gamma_{a} \nabla_{e_{a}}+\psi_{A}
$$

for some endomorphism $\psi_{A}$ of $\left.V\right|_{\partial M} ; A$ is of Dirac type on $\left.V\right|_{\partial M}$ with respect to the induced tangential Clifford module structure $\gamma_{a}^{T}:=-\gamma_{m} \gamma_{a}$.

For the sake of simplicity, we shall assume $A$ has no purely imaginary eigenvalues. Let $\Pi_{A}^{+}$be spectral projection on the span of the generalized eigenspaces of $A$ corresponding to eigenvalues with positive real part. This spectral projection defines a boundary condition for $P$; the associated boundary operator for $D$ is

$$
\mathcal{B}:=\Pi_{A}^{+} \oplus \Pi_{A}^{+} P .
$$

Let $D_{\mathcal{B}}$ be the associated realization. The fundamental solution $e^{-t D_{\mathcal{B}}}$ of the heat equation is well defined; $u=e^{-t D_{\mathcal{B}}} \phi$ is characterized by the relations

$$
\left(\partial_{t}+D\right) u=0, \quad \mathcal{B} u=0, \quad \text { and }\left.u\right|_{t=0}=\phi .
$$

We refer to Grubb [10, 11] and Grubb and Seeley [12, 13, 14 for further details.

Let $\langle\cdot, \cdot\rangle$ be the natural pairing between $V$ and the dual bundle $V^{*}$, let $d x$ be the Riemannian measure on $M$, and let $\rho \in C^{\infty}\left(V^{*}\right)$ be the specific heat. The total heat energy content of the manifold with initial temperature $\phi$ is given by

$$
\beta(\phi, \rho, D, \mathcal{B})(t):=\int_{M}\langle u(x ; t), \rho(x)\rangle d x .
$$

If we had imposed local boundary conditions such as Dirichlet or Robin, then it is well known that there is a complete asymptotic series for $\beta$ with locally computable

2000 Mathematics Subject Classification. Primary 58J50.

Key words and phrases. Laplace type, Dirac type, heat content asymptotics, spectral boundary conditions. 
coefficients $\beta_{n}$. We refer to [2, 3, 4, 8, 15, 16, 17, 18, for a discussion of this case. Thus we assume there exists a complete asymptotic series as $t \downarrow 0$ of the form

$$
\beta(\phi, \rho, D, \mathcal{B})(t) \sim \sum_{n=0}^{\infty} \beta_{n}(\phi, \rho, D, \mathcal{B}) t^{n / 2} .
$$

If $\Psi$ is an operator on $C^{\infty}(V)$ or on $C^{\infty}\left(V^{*}\right)$, let $\tilde{\Psi}$ be the formal adjoint on $C^{\infty}\left(V^{*}\right)$ or on $C^{\infty}(V)$, respectively. Similarly, if $\Psi$ is an operator on $C^{\infty}\left(\left.V\right|_{\partial M}\right)$ or on $C^{\infty}\left(\left.V^{*}\right|_{\partial M}\right)$, let $\tilde{\Psi}$ be the adjoint on $C^{\infty}\left(\left.V^{*}\right|_{\partial M}\right)$ or on $C^{\infty}\left(\left.V\right|_{\partial M}\right)$, respectively. Let $\tilde{\nabla}$ be the dual connection on $V^{*}$, let $L_{a b}$ be the second fundamental form of the boundary, and let $d y$ be the Riemannian measure on the boundary. We omit the proof of the following Lemma in the interests of brevity as it is straightforward.

Lemma 1.1. (1) $\tilde{P}=-\tilde{\gamma}_{i} \tilde{\nabla}_{e_{i}}+\tilde{\psi}_{P}$ and $\tilde{A}=-\tilde{\gamma}_{m} \tilde{\gamma}_{a} \tilde{\nabla}_{e_{a}}+\tilde{\psi}_{A}$.

(2) Let $A^{\#}:=\tilde{\gamma}_{m} \tilde{A} \tilde{\gamma}_{m}$ on $C^{\infty}\left(\left.V^{*}\right|_{\partial M}\right)$. Then:

(a) $\Pi_{A^{\#}}^{+}$defines the adjoint boundary condition for $\tilde{P}$.

(b) $\tilde{B}:=\Pi_{A^{\#}}^{+} \oplus \Pi_{A_{\sim}^{\#}}^{+} \tilde{P}$ defines the adjoint boundary condition for $\tilde{D}:=\tilde{P}^{2}$.

(c) Let $\psi_{A^{\#}}:=\tilde{\gamma}_{m} \tilde{\psi}_{A} \tilde{\gamma}_{m}+L_{a a}$ Id. Then $A^{\#}=-\tilde{\gamma}_{m} \tilde{\gamma}_{a} \tilde{\nabla}_{e_{a}}+\psi_{A \#}$.

(3) $\int_{M}\{\langle D \phi, \rho\rangle-\langle\phi, \tilde{D} \rho\rangle\} d x=-\int_{\partial M}\left\{\left\langle\gamma_{m} \Pi_{A}^{+} P \phi, \rho\right\rangle+\left\langle P \phi, \tilde{\gamma}_{m} \Pi_{A^{\#}}^{+} \rho\right\rangle\right.$ $\left.+\left\langle\phi, \tilde{\gamma}_{m} \Pi_{A^{\#}}^{+} \tilde{P} \rho\right\rangle+\left\langle\gamma_{m} \Pi_{A}^{+} \phi, \tilde{P} \rho\right\rangle\right\} d y$.

(4) We have $\left(\mathrm{Id}-\widetilde{\Pi_{A}^{+}}\right) \tilde{\gamma}_{m}=\tilde{\gamma}_{m} \Pi_{A^{\#}}^{+}$.

(5) If $\mathcal{B} \phi=0$ and if $\tilde{\mathcal{B}} \rho=0$, then $\int_{M}\{\langle D \phi, \rho\rangle-\langle\phi, \tilde{D} \rho\rangle\} d x=0$.

We can now state the main result of this paper:

Theorem 1.2. Adopt the notation established above.

(1) $\beta_{0}(\phi, \rho, D, \mathcal{B})=\int_{M}\langle\phi, \rho\rangle d x$.

(2) $\beta_{1}(\phi, \rho, D, \mathcal{B})=-\frac{2}{\sqrt{\pi}} \int_{\partial M}\left\langle\Pi_{A}^{+} \phi, \Pi_{A \#}^{+} \rho\right\rangle d y$.

(3) $\beta_{2}(\phi, \rho, D, \mathcal{B})=-\int_{M}\langle D \phi, \rho\rangle d x+\int_{\partial M}\left\{-\left\langle\gamma_{m} \Pi_{A}^{+} P \phi, \rho\right\rangle-\left\langle\gamma_{m} \Pi_{A}^{+} \phi, \tilde{P} \rho\right\rangle\right.$ $\left.+\frac{1}{2}\left\langle\left(L_{a a}+A+\widetilde{A^{\#}}-\gamma_{m} \psi_{P}+\psi_{P} \gamma_{m}-\psi_{A}-\widetilde{\psi_{A \#}}\right) \Pi_{A}^{+} \phi, \Pi_{A^{\#}}^{+} \rho\right\rangle\right\} d y$.

A variant of this result was established in [9] using a special case calculation. In this paper, we extend this result by using functorial methods. In Section 2 we derive various naturality properties of these invariants. These properties are then used in Section [3] to complete the proof.

\section{Properties of the heat Content invariants}

We begin with some general observations:

Lemma 2.1. (1) $\beta_{0}(\phi, \rho, D, \mathcal{B})=\int_{M}\langle\phi, \rho\rangle d x$.

(2) $\beta_{n}(\phi, \rho, D, \mathcal{B})=\beta_{n}(\rho, \phi, \tilde{D}, \tilde{\mathcal{B}})$.

(3) If $\mathcal{B} \phi=0$, then $\frac{n}{2} \beta_{n}(\phi, \rho, D, \mathcal{B})=-\beta_{n-2}(D \phi, \rho, D, \mathcal{B})$.

(4) If $M$ is closed, then $\beta_{n}(\phi, \rho, D, \mathcal{B})=0$ if $n$ is odd while if $n=2 k$ is even, then $\beta_{2 k}(\phi, \rho, D, \mathcal{B})=(-1)^{k} \frac{1}{k !} \int_{M}\left\langle D^{k} \phi, \rho\right\rangle d x$.

(5) $\beta_{n}\left(\phi, \rho,(-P)^{2}, \mathcal{B}\right)=\beta_{n}\left(\phi, \rho, P^{2}, \mathcal{B}\right)$.

Proof. The first assertion is immediate since $\left.u\right|_{t=0}=\phi$. To prove the second assertion, we set $u(x ; t):=e^{-t D_{\mathcal{B}}} \phi$ and $\tilde{u}(x ; t):=e^{-t \tilde{D}_{\tilde{\mathcal{B}}} \rho}$. We then have

$$
\partial_{t} u=-D u \quad \text { and } \quad \partial_{t} \tilde{u}=-\tilde{D} \tilde{u} \text {. }
$$

Fix $t>0$. For $0 \leq s \leq t$, set $f(s):=\int_{M}\langle u(x ; s), \tilde{u}(x ; t-s)\rangle d x$. By Lemma 1.1 (5),

$$
\begin{aligned}
\partial_{s} f(s) & =\int_{M}\left\{\left\langle\partial_{s} u(x ; s), \tilde{u}(x ; t-s)\right\rangle-\left\langle u(x ; s), \partial_{t} \tilde{u}(x ; t-s)\right\rangle\right\} d x \\
& =\int_{M}\{-\langle D u(x ; s), \tilde{u}(x ; t-s)\rangle+\langle u(x ; s), \tilde{D} \tilde{u}(x ; t-s)\rangle\} d x \\
& =0
\end{aligned}
$$


since $\mathcal{B} u=0$ and $\tilde{\mathcal{B}} \tilde{u}=0$. Because $f(s)$ is constant, Assertion (2) follows as

$$
\begin{aligned}
0 & =f(t)-f(0)=\int_{M}\{\langle u(x ; t), \tilde{u}(x ; 0)\rangle-\langle u(x ; 0), \tilde{u}(x ; t)\rangle\} d x \\
& =\beta(\phi, \rho, D, \mathcal{B})(t)-\beta(\rho, \phi, \tilde{D}, \tilde{\mathcal{B}})(t) .
\end{aligned}
$$

To establish the third assertion, we suppose that $\mathcal{B} \phi=0$. Since $\tilde{\mathcal{B}} \tilde{u}=0$, we can use Lemma 1.1 (5) and Assertion (2) to see

$$
\begin{aligned}
& \partial_{t} \beta(\phi, \rho, D, \mathcal{B})(t)=\partial_{t} \beta(\rho, \phi, \tilde{D}, \tilde{\mathcal{B}})(t) \\
= & \int_{M}\left\langle\partial_{t} \tilde{u}(x ; t), \phi(x)\right\rangle d x=-\int_{M}\langle\tilde{D} \tilde{u}(x ; t), \phi(x)\rangle d x \\
= & -\int_{M}\langle\tilde{u}(x ; t), D \phi(x)\rangle d x=-\beta(\rho, D \phi, \tilde{D}, \tilde{\mathcal{B}})(t) \\
= & -\beta(D \phi, \rho, D, \mathcal{B})(t) .
\end{aligned}
$$

We equate terms in the asymptotic expansions to derive Assertion (3). Assertion (4) follows by induction from Assertions (1) and (3); Assertion (5) is immediate.

Mixed boundary conditions will play an important role in our discussion. Assume given an endomorphism $\gamma_{0}$ of $\left.V\right|_{\partial M}$ so that $\gamma_{0}^{2}=$ Id. Let

$$
\Xi_{ \pm}:=\frac{1}{2}\left(\operatorname{Id} \pm \gamma_{0}\right) \text { and } V_{ \pm}:=\Xi_{ \pm}\left\{\left.V\right|_{\partial M}\right\}
$$

be the associated spectral projections and eigenspaces. For $S \in \operatorname{End}\left(\left.V\right|_{\partial M}\right)$, set

$$
\mathcal{B}_{\gamma_{0}, S} \phi:=\left.\left.\Xi_{-}\left(\nabla_{e_{m}}+S\right) \phi\right|_{\partial M} \oplus \Xi_{+} \phi\right|_{\partial M}
$$

The operator $D$ determines a natural connection $\nabla^{D}$. The following Theorem, after taking into account our sign conventions, follows from results of 4 .

Theorem 2.2. Adopt the notation established above. Then:

(1) $\beta_{0}\left(\phi, \rho, D, \mathcal{B}_{\gamma_{0}, S}\right)=\int_{M}\langle\phi, \rho\rangle d x$.

(2) $\beta_{1}\left(\phi, \rho, D, \mathcal{B}_{\gamma_{0}, S}\right)=-\frac{2}{\sqrt{\pi}} \int_{\partial M}\left\langle\Xi_{+} \phi, \tilde{\Xi}_{+} \rho\right\rangle d y$.

(3) $\beta_{2}\left(\phi, \rho, D, \mathcal{B}_{\gamma_{0}, S}\right)=-\int_{M}\langle D \phi, \rho\rangle d x+\int_{\partial M}\left\{\left\langle\Xi_{-}\left(\nabla_{e_{m}}^{D} \phi+S \phi\right), \rho\right\rangle\right.$

$$
\left.+\left\langle\frac{1}{2} L_{a a} \Xi_{+} \phi, \rho\right\rangle-\left\langle\Xi_{+} \phi, \tilde{\nabla}_{e_{m}}^{D} \rho\right\rangle\right\} d y \text {. }
$$

Example 2.3. We can relate spectral and mixed boundary conditions in the following special setting. Let $\left(\theta_{1}, \ldots, \theta_{m-1}\right)$ be the usual periodic parameters on the torus $\mathbb{T}^{m-1}$ and let $r$ be the radial parameter on the interval $[0,1]$. Let $f \in C^{\infty}[0,1]$ with $f(0)=f(1)=0$. Take a warped product on $M:=\mathbb{T}^{m-1} \times[0,1]$ of the form:

$$
d s_{M}^{2}:=e^{2 f(r)} d \theta^{a} \circ d \theta^{a}+d r^{2} .
$$

The volume element is then given by $d x=g d r d \theta_{1} \ldots d \theta_{m-1}$ where $g:=e^{(m-1) f}$. Let $\varepsilon(0):=+1$ and $\varepsilon(1):=-1$ so that the inward unit normal is $\varepsilon \partial_{r}$. Let $\Theta_{i} \in M_{\ell}(\mathbb{C})$ satisfy the Clifford commutation relations $\Theta_{i} \Theta_{j}+\Theta_{j} \Theta_{i}=-2 \delta_{i j}$. Set

$$
\gamma_{m}:=\Theta_{m}, \quad \gamma_{a}:=e^{f} \Theta_{a}, \quad \text { and } \quad \gamma^{a}:=e^{-f} \Theta_{a} .
$$

Let $\gamma_{0} \in M_{\ell}(\mathbb{C})$ satisfy $\gamma_{0}^{2}=\mathrm{Id}$ and $\gamma_{0} \gamma_{m}+\gamma_{m} \gamma_{0}=0$. Let $\delta_{1}>0$ and $\delta_{2}>0$ be real parameters. Let $V=M \times \mathbb{C}^{\ell}$, let $\phi=\phi(r)$ and let $\rho=\rho(r)$. Set

$$
\begin{array}{lll}
P:=\gamma_{m} \partial_{r}+\gamma_{a} \partial_{a}^{\theta}+\delta_{1} \gamma_{m} \gamma_{0} & \text { on } & C^{\infty}(V), \\
A:=-\varepsilon \gamma_{m} \gamma_{a} \partial_{a}^{\theta}+\delta_{2} \gamma_{0} & \text { on } & C^{\infty}\left(\left.V\right|_{\partial M}\right) .
\end{array}
$$

For generic values of $\delta_{2}, \operatorname{ker}(A)=\{0\}$.

Let $V_{0}:=[0,1] \times \mathbb{C}^{\ell}$. Let $\Xi_{ \pm}$be projection on the \pm 1 eigenspaces of $\gamma_{0}$; we have that $\gamma_{m} \Xi_{-}=\Xi_{+} \gamma_{m}$. Let $P_{0}:=\gamma_{m} \partial_{r}+\delta_{1} \gamma_{m} \gamma_{0}$ on $C^{\infty}\left(V_{0}\right)$, and let $D_{0}:=P_{0}^{2}$. Set

$$
\mathcal{B}_{0} \phi:=\left.\left\{\Xi_{+} \phi \oplus \Xi_{+} P_{0} \phi\right\}\right|_{\partial[0,1]} \text { and } S:=\delta_{1} \gamma_{0} \text {. }
$$


We show that $\mathcal{B}_{0}$ and $\mathcal{B}_{\gamma_{0}, S}$ define the same boundary conditions for $D_{0}$ by checking:

$$
\begin{aligned}
\Xi_{+}\left(\left.\phi\right|_{\partial[0,1]}\right)=0 \quad \text { and }\left.\quad \Xi_{+} P_{0} \phi\right|_{\partial[0,1]}=0, \\
\Leftrightarrow \quad \Xi_{+}\left(\left.\phi\right|_{\partial[0,1]}\right)=0 \quad \text { and }\left.\quad \Xi_{+} \gamma_{m}\left(\partial_{r} \phi+\delta_{1} \gamma_{0} \phi\right)\right|_{\partial[0,1]}=0, \\
\Leftrightarrow \quad \Xi_{+}\left(\left.\phi\right|_{\partial[0,1]}\right)=0 \quad \text { and }\left.\quad \gamma_{m} \Xi_{-}\left(\partial_{r} \phi+\delta_{1} \gamma_{0} \phi\right)\right|_{\partial[0,1]}=0, \\
\Leftrightarrow \quad \Xi_{+}\left(\left.\phi\right|_{\partial[0,1]}\right)=0 \quad \text { and }\left.\quad \Xi_{-}\left(\partial_{r}+S\right) \phi\right|_{\partial[0,1]}=0 .
\end{aligned}
$$

Lemma 2.4. $\beta_{n}\left(\phi, g^{-1} \rho, D, \mathcal{B}\right)=(2 \pi)^{m-1} \beta_{n}\left(\phi, \rho, D_{0}, \mathcal{B}_{\gamma_{0}, S}\right)$ in Example 2.3

Proof. Let $u_{0}:=e^{-t D_{0, \mathcal{B}_{0}} \phi}$. Set $u(r, \theta ; t):=u_{0}(r ; t)$. If $\Phi=\Phi(r)$ and $\Psi=\Psi(r)$, then $\Pi_{A}^{+} \Phi=\Xi_{+} \Phi$ and $\Pi_{A \#}^{+} \Psi=\tilde{\Xi}_{+} \Psi$. Thus we may show $u=e^{-t D_{\mathcal{B}} \phi}$ by checking

$$
\begin{aligned}
& \left(\partial_{t}+D\right) u=\left(\partial_{t}+D_{0}\right) u=0, \\
& \mathcal{B} u=\Pi_{A}^{+} u \oplus \Pi_{A}^{+} P u=\Xi_{+} u \oplus \Xi_{+} P_{0} u=\mathcal{B}_{0} u_{0}=0, \\
& \left.u\right|_{t=0}=\left.u_{0}\right|_{t=0}=\phi .
\end{aligned}
$$

After taking into account the change in volume elements and equating terms in the asymptotic expansions, the result follows.

Remark 2.5. The coefficients appearing in Theorem 1.2 are independent of the dimension $m$. This observation is quite general. Let $P_{0}$ be an operator of Dirac type on a bundle $V_{0}$ over an $m$ dimensional manifold $M_{0}$. By doubling the rank of $V_{0}$ and by replacing $P_{0}$ by $P_{0} \oplus-P_{0}$ if necessary, we may suppose there exists $\gamma_{0}$ so

$$
\gamma_{0} P_{0}+P_{0} \gamma_{0}=0 \text { and } \gamma_{0}^{2}=-\mathrm{Id} \text {. }
$$

Define analogous structures on $M:=M_{0} \times S^{1}$ by setting

$$
P:=P_{0}+\gamma_{0} \partial_{\theta} \quad \text { and } \quad A:=A_{0}-\gamma_{m} \gamma_{0} \partial_{\theta} .
$$

Let $\phi(x, \theta)=\phi_{0}(x)$ and let $u_{0}:=e^{-t D_{0, \mathcal{B}_{0}}} \phi_{0}$. Set $u(x, \theta ; t):=u_{0}(x ; t)$. Then one

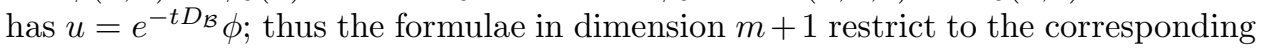
formulae in dimension $m$. By contrast, the corresponding formulae for the heat trace asymptotics [5, 7] exhibit a very complicated dependence on $m$.

\section{Proof of Theorem 1.2}

Since $\left.u\right|_{t=0}=\phi$, Assertion (1) of Theorem 1.2 is immediate. The interior integrands in $\beta_{1}$ and $\beta_{2}$ are determined by Lemma 2.1 (4). Thus we need only determine the boundary integrands. We apply Lemma 2.1 throughout. Dimensional analysis shows that the boundary integrands are homogeneous of total weight $n-1$ in the jets of $\phi$, of $\rho$, and of the derivatives of the symbols of $A$ and of $P$. The spectral projections $\Pi_{A}^{+}$and $\Pi_{A^{\#}}^{+}$and the endomorphisms $\gamma_{i}$ have weight 0 ; the second fundamental form $L$, the operators $A$ and $P$, and the endomorphisms $\psi_{A}$ and $\psi_{P}$ have weight 1.

We begin by studying $\beta_{1}$; there is no interior contribution. If $\mathcal{B} \phi=0$, then by Lemma $2.1(3), \beta_{1}(\phi, \rho, D, \mathcal{B})=0$. Dually by Lemma 2.1 $(2)$,

$$
\beta_{1}(\phi, \rho, D, \mathcal{B})=\beta_{1}(\rho, \phi, \tilde{D}, \tilde{\mathcal{B}})=0 \text { if } \tilde{\mathcal{B}} \rho=0 \text {. }
$$

Since the boundary integrand for $\beta_{1}$ must be homogeneous of weight 0 , there exist universal constants so

$$
\beta_{1}(\phi, \rho, D, \mathcal{B})=\int_{\partial M}\left\{c_{0}(m)\left\langle\Pi_{A}^{+} \phi, \Pi_{A \#}^{+} \rho\right\rangle+c_{1}(m)\left\langle\gamma_{m} \Pi_{A}^{+} \phi, \Pi_{A \#}^{+} \rho\right\rangle\right\} d y .
$$

By Lemma 2.1 $(5), \beta_{1}\left(\phi, \rho, P^{2}, \mathcal{B}\right)=\beta_{1}\left(\phi, \rho,(-P)^{2}, \mathcal{B}\right)$. Replacing $P$ by $-P$ replaces $\gamma_{m}$ by $-\gamma_{m}$. Thus $\left\langle\gamma_{m} \Pi_{A}^{+} \phi, \Pi_{A \#}^{+} \rho\right\rangle$ plays no role so we may take $c_{1}(m)=0$.

We apply Lemma 2.4 with $f=0$. By Theorem 2.2 and Equation (3.a),

$$
\begin{aligned}
\beta_{1}(\phi, \rho, D, \mathcal{B}) & =(2 \pi)^{m-1} \beta_{1}\left(\phi, \rho, D_{0}, \mathcal{B}_{0}\right)=-\frac{2}{\sqrt{\pi}} \int_{\partial M}\left\langle\Xi_{+} \phi, \tilde{\Xi}_{+} \rho\right\rangle d y \\
& =c_{0}(m) \int_{\partial M}\left\langle\Xi_{+} \phi, \tilde{\Xi}_{+} \rho\right\rangle d y
\end{aligned}
$$


This shows that $c_{0}(m)=-\frac{2}{\sqrt{\pi}}$ which completes the proof of Theorem $1.2(2)$.

Remark 3.1. The constant $c_{0}(m)$ was determined in 9 using a special case computation and the present calculation should be regarded as providing a useful cross check on that calculation.

To prove the final assertion of Theorem 1.2 we express $\beta_{2}$ in terms of invariants with undetermined universal coefficients.

Lemma 3.2. There exist universal constants $c_{i}$ so that

$$
\begin{aligned}
& \beta_{2}(\phi, \rho, D, \mathcal{B})=-\int_{M}\langle D \phi, \rho\rangle d x+\int_{\partial M}\left\{-\left\langle\gamma_{m} \Pi_{A}^{+} P \phi, \rho\right\rangle\right. \\
& \quad-\left\langle\gamma_{m} \Pi_{A}^{+} \phi, \tilde{P} \rho\right\rangle+\left\langle\left( c_{2}\left(A+\widetilde{A^{\#}}\right)+c_{3} L_{a a}+c_{4}\left(\gamma_{m} \psi_{P}-\psi_{P} \gamma_{m}\right)\right.\right. \\
& \left.\left.\left.\quad+c_{5}\left(\psi_{A}+\widetilde{\psi_{A^{\#}}}\right)\right) \Pi_{A}^{+} \phi, \Pi_{A^{\#}}^{+} \rho\right\rangle\right\} d y .
\end{aligned}
$$

Proof. We argue heuristically. By Lemma 2.1 the interior integral for $\beta_{2}$ is given by $-\langle D \phi, \rho\rangle$. We define the normalized invariant $\mathcal{C}$, which is given by a suitable boundary integral, by the identity

$$
\begin{aligned}
\beta_{2}(\phi, \rho, D, \mathcal{B})= & \mathcal{C}(\phi, \rho, D, \mathcal{B})-\int_{M}\langle D \phi, \rho\rangle d x \\
& +\int_{\partial M}\left\{-\left\langle\gamma_{m} \Pi_{A}^{+} P \phi, \rho\right\rangle-\left\langle\gamma_{m} \Pi_{A}^{+} \phi, \tilde{P} \rho\right\rangle\right\} d y .
\end{aligned}
$$

As we must replace $\gamma_{m}$ by $-\tilde{\gamma}_{m}$ in passing to the dual structures, we have

$$
\begin{aligned}
0= & \beta_{2}(\phi, \rho, D, \mathcal{B})-\beta_{2}(\rho, \phi, \tilde{D}, \tilde{\mathcal{B}}) \\
= & \mathcal{C}(\phi, \rho, D, \mathcal{B})-\mathcal{C}(\rho, \phi, \tilde{D}, \tilde{\mathcal{B}})-\int_{M}\{\langle D \phi, \rho\rangle-\langle\phi, \tilde{D} \rho\rangle\} d x \\
& +\int_{\partial M}\left\{-\left\langle\gamma_{m} \Pi_{A}^{+} P \phi, \rho\right\rangle-\left\langle\gamma_{m} \Pi_{A}^{+} \phi, \tilde{P} \rho\right\rangle-\left\langle\phi, \tilde{\gamma}_{m} \Pi_{A^{\#}}^{+} \tilde{P} \rho\right\rangle\right. \\
& \left.\quad-\left\langle P \phi, \tilde{\gamma}_{m} \Pi_{A^{\#}}^{+} \rho\right\rangle\right\} d y .
\end{aligned}
$$

We now use the Greens formula given in Lemma 1.1 (3) to see that

$$
\mathcal{C}(\phi, \rho, D, \mathcal{B})=\mathcal{C}(\rho, \phi, \tilde{D}, \tilde{\mathcal{B}})
$$

Thus we can assume that the integral expressions for $\mathcal{C}$ are symmetric in $\phi$ and $\rho$.

If $\mathcal{B} \phi=0$, then $\mathcal{C}(\phi, \rho, D, \mathcal{B})=0$ by Lemma 2.1 (3). Similarly $\mathcal{C}(\phi, \rho, D, \mathcal{B})=0$ if $\tilde{\mathcal{B}} \rho=0$. Thus after eliminating divergence terms, the integral formula for $\mathcal{C}$ is bilinearly expressible in terms of tangential operators applied to

$$
\left\{\Pi_{A}^{+} \phi, \Pi_{A}^{+} P \phi\right\} \quad \text { and } \quad\left\{\Pi_{A^{\#}}^{+} \rho, \Pi_{A^{\#}}^{+} \tilde{P} \rho\right\} .
$$

Since the boundary integrals defining $\mathcal{C}$ have total weight 1 , terms which are bilinear in $\Pi_{A}^{+} P \phi$ and $\Pi_{A^{\#}}^{+} \tilde{P} \rho$ do not appear. By Lemma 1.1 (4),

$$
\gamma_{m} \Pi_{A}^{+}=\left(\operatorname{Id}-\tilde{\Pi}_{A^{\#}}^{+}\right) \gamma_{m} \quad \text { so } \quad \int_{\partial M}\left\langle\gamma_{m} \Pi_{A}^{+} \Phi, \Pi_{A^{\#}}^{+} \tilde{\Phi}\right\rangle=0
$$

for any $\Phi, \tilde{\Phi}$. This shows that terms which are bilinear in $\Pi_{A}^{+} P \phi$ and $\Pi_{A^{*}}^{+} \rho$ or in $\Pi_{A}^{+} \phi$ and $\Pi_{A^{\#}}^{+} \tilde{P} \rho$ do not involve $\gamma_{m}$. Taking into account the symmetry of $\mathcal{C}$, we see that these terms would have the form

$$
\int_{\partial M} b_{0}\left(\left\langle\Pi_{A}^{+} P \phi, \Pi_{A \#}^{+} \rho\right\rangle+\left\langle\Pi_{A}^{+} \phi, \Pi_{A^{\#}}^{+} \tilde{P} \rho\right\rangle\right) d y .
$$

Lemma $2.1(5)$ now shows $b_{0}=0$. Consequently

$$
\begin{aligned}
& \mathcal{C}(\phi, \rho, D, \mathcal{B})=\int_{\partial M}\left\langle\mathcal{T} \Pi_{A}^{+} \phi, \Pi_{A^{\#}}^{+} \rho\right\rangle d y \quad \text { where } \\
& \mathcal{T}=b_{1} A+b_{2} \gamma_{m} A+b_{3} A \gamma_{m}+b_{4} \gamma_{m} A \gamma_{m} \\
& \quad+c_{3} L_{a a} \mathrm{Id}+b_{5} \psi_{P}+b_{6} \gamma_{m} \psi_{P}+b_{7} \psi_{P} \gamma_{m}+b_{8} \gamma_{m} \psi_{P} \gamma_{m} \\
& \quad+b_{9} \psi_{A}+b_{10} \gamma_{m} \psi_{A}+b_{11} \widetilde{\psi_{A^{\#}}} \gamma_{m}+b_{12} \widetilde{\psi_{A^{\#}}}
\end{aligned}
$$

It is worth while making a few remarks about what invariants do not appear. Modulo terms in $L_{a a}$, we can replace $\gamma_{m} \psi_{A} \gamma_{m}$ by $\widetilde{\psi_{A^{\#}}}$ and $\psi_{A}$ by $-\widetilde{\psi_{A} \#} \gamma_{m}$. By Lemma 
2.1 (5), $\gamma_{m} L_{a a}$ can not appear. Furthermore, the invariants $\gamma_{a} \psi_{P} \gamma_{a}$ and $\gamma_{a} \gamma_{m} \psi_{P} \gamma_{a}$ would violate Remark 2.5.

Replacing $P$ by $\tilde{P}$ replaces $\gamma_{m}$ by $-\tilde{\gamma}_{m}, A$ by $A^{\#}, \psi_{P}$ by $\tilde{\psi}_{P}$, and $\psi_{A}$ by $\psi_{A^{\#}}$. Thus the symmetry of Lemma 2.1 (2) yields $b_{1}=b_{4}, b_{6}=-b_{7}$, and $b_{9}=b_{12}$. Lemma 2.1 (5) implies $b_{2}=b_{3}=b_{5}=b_{8}=b_{10}=b_{11}=0$. Setting $b_{1}=b_{4}=c_{2}$, $b_{6}=-b_{7}=c_{4}$, and $b_{9}=b_{12}=c_{5}$ then yields the formula of the Lemma; we use Remark 2.5 to see the coefficients $c_{i}$ are universal.

We complete the proof of Theorem 1.2 (3) by showing:

Lemma 3.3.

(2) $c_{3}=\frac{1}{2}$.

(1) $c_{2}=\frac{1}{2}, c_{4}=-\frac{1}{2}, c_{5}=-\frac{1}{2}$.

Proof. Again, we take the flat metric in Example 2.3 The flat connection is compatible with the Clifford module structure. It is not, however, the only possible compatible connection. Let $\varrho_{a}$ be auxiliary real constants. We define a compatible connection by setting $\omega_{a}:=\varrho_{a}$ Id. As $\gamma_{a}$ and $\gamma_{0}$ anti-commute with $\gamma_{m}$,

$$
\begin{array}{lll}
\psi_{P}=\delta_{1} \gamma_{m} \gamma_{0}-\varrho_{a} \gamma_{a} & \text { so } \quad \gamma_{m} \psi_{P}-\psi_{P} \gamma_{m}=-2 \delta_{1} \gamma_{0}-2 \gamma_{m} \gamma_{a} \varrho_{a} \\
\psi_{A}=\delta_{2} \gamma_{0}+\varepsilon \gamma_{m} \gamma_{a} \varrho_{a} & \text { so } \quad \psi_{A}+\gamma_{m} \psi_{A} \gamma_{m}=2 \delta_{2} \gamma_{0}+2 \varepsilon \gamma_{m} \gamma_{a} \varrho_{a}
\end{array}
$$

We take $\rho=0$ near $r=1$ so only the component where $r=0$ is relevant in integrating over $\partial M$. On this component, $\partial_{r}$ is the inward geodesic normal and we set $\varepsilon=1$. By Lemma 3.2

$$
\begin{aligned}
& \beta_{2}(\phi, \rho, D, \mathcal{B})=-\int_{M}\langle D \phi, \rho\rangle+\int_{\partial M}\left\{-\left\langle\gamma_{m} \Xi_{+} \gamma_{m}\left(\partial_{r}+\delta_{1} \gamma_{0}\right) \phi, \rho\right\rangle\right. \\
+ & \left\langle\gamma_{m} \Xi_{+} \phi, \tilde{\gamma}_{m}\left(\partial_{r}+\delta_{1} \tilde{\gamma}_{0}\right) \rho\right\rangle+\left\langle\left(\left(2 c_{2}+2 c_{5}\right) \delta_{2} \gamma_{0}\right.\right. \\
+ & \left.\left.\left.\left(2 c_{5}-2 c_{4}\right) \gamma_{m} \gamma_{a} \varrho_{a}-2 c_{4} \delta_{1} \gamma_{0}\right) \Xi_{+} \phi, \tilde{\Xi}_{+} \rho\right\rangle\right\} d y \\
= & -\int_{M}\langle D \phi, \rho\rangle+\int_{\partial M}\left\{\left\langle\Xi_{-}\left(\partial_{r}+\delta_{1} \gamma_{0}\right) \phi, \rho\right\rangle-\left\langle\Xi_{+} \phi, \partial_{r} \rho\right\rangle\right. \\
+ & \left.\left\langle\left[\left(-1-2 c_{4}\right) \delta_{1} \gamma_{0}+\left(2 c_{2}+2 c_{5}\right) \delta_{2} \gamma_{0}+\left(2 c_{5}-2 c_{4}\right) \gamma_{m} \gamma_{a} \varrho_{a}\right] \Xi_{+} \phi, \rho\right\rangle\right\} d y .
\end{aligned}
$$

On the other hand, since $P_{0}^{2}=-\left(\partial_{r}^{2}-\delta_{1}^{2}\right) \mathrm{Id}$, the connection defined by $D_{0}$ is the trivial connection. Thus by Theorem 2.2

$$
\begin{aligned}
& (2 \pi)^{m-1} \beta_{2}\left(\phi, \rho, D_{0}, \mathcal{B}_{0}\right) \\
= & -\int_{M}\langle D \phi, \rho\rangle d x+\int_{\partial M}\left\{\left\langle\Xi_{-}\left(\partial_{r}+\delta_{1} \gamma_{0}\right) \phi, \rho\right\rangle-\left\langle\Xi_{+} \phi, \partial_{r} \rho\right\rangle\right\} d y .
\end{aligned}
$$

By Lemma 2.4 $\beta_{2}(\phi, \rho, D, \mathcal{B})=(2 \pi)^{m-1} \beta_{2}\left(\phi, \rho, D_{0}, \mathcal{B}_{0}\right)$. We may now complete the proof of Assertion (1) by deriving the relations

$$
2 c_{2}+2 c_{5}=0, \quad 2 c_{5}-2 c_{4}=0, \quad \text { and } \quad 2 c_{4}=-1 .
$$

To study the coefficient of $L_{a a}$, we let $f$ be arbitrary in Example 2.3 but set $\delta_{1}=0$. We have $\widetilde{A^{\#}}=A$; thus $\tilde{\Pi}_{A^{\#}}^{+}=\Pi_{A}^{+}$. Let $\phi=\phi(r)$ and $\rho=\rho(r)$. We suppose $\rho$ vanishes identically near $r=1$ and suppress $\varepsilon$. We may then apply Lemma 2.4 and Theorem 2.2 with $S=0$ to compute:

$$
\begin{aligned}
& \beta_{2}\left(\phi, g^{-1} \rho, D, \mathcal{B}\right)=(2 \pi)^{m-1} \beta_{2}\left(\phi, \rho, D_{0}, \mathcal{B}_{0}\right) \\
= & \int_{M}-\left\langle D_{0} \phi, \rho\right\rangle d r d \theta+\int_{\partial M}\left\{\left\langle\Xi_{-} \partial_{r} \phi, \rho\right\rangle-\left\langle\Xi_{+} \phi, \partial_{r} \rho\right\rangle\right\} d \theta .
\end{aligned}
$$

We have $\Pi_{A}^{+}=\Xi_{+}$and $\Pi_{A^{\#}}^{+}=\tilde{\Xi}_{+}$. Since $\tilde{P} g^{-1} \rho=-g^{-1} \tilde{\gamma}_{m} \partial_{r} \rho$ and $d y=g d \theta$,

$$
\begin{aligned}
& \int_{\partial M}\left\{\left\langle\Xi_{-} \partial_{r} \phi, \rho\right\rangle-\left\langle\Xi_{+} \phi, \partial_{r} \rho\right\rangle\right\} d \theta \\
= & \int_{\partial M}\left\{-\left\langle\gamma_{m} \Pi_{A}^{+} P \phi, g^{-1} \rho\right\rangle-\left\langle\gamma_{m} \Pi_{A}^{+} \phi, \tilde{P} g^{-1} \rho\right\rangle\right\} d y .
\end{aligned}
$$

Consequently we have

$$
0=\left\{c_{2}\left(A+A^{\#}\right)+c_{3} L_{a a}+c_{4}\left(\gamma_{m} \psi_{P}-\psi_{P} \gamma_{m}\right)+c_{5}\left(\psi_{A}+\widetilde{\psi_{A_{\#}}}\right)\right\} \Pi_{A}^{+} \phi .
$$


We must define a compatible connection. Let $\omega_{m}=0$ and $\omega_{a}=\frac{1}{2} \partial_{r} f \cdot \gamma_{m} \gamma_{a}$ define a connection $\nabla$ on $V$. We have

$$
\begin{aligned}
\Gamma_{m a b} & =\Gamma_{a m b}=-\Gamma_{a b m}=\delta_{a b} e^{2 f} \partial_{r} f, \\
\Gamma_{m a}{ }^{b} & =\Gamma_{a m}{ }^{b}=\delta_{a b} \partial_{r} f, \quad \text { and } \quad \Gamma_{a b}{ }^{m}=-\delta_{a b} e^{2 f} \partial_{r} f .
\end{aligned}
$$

We have $\gamma_{j ; i}=\partial_{i}^{x} \gamma_{j}-\Gamma_{i j}{ }^{k} \gamma_{k}+\left[\omega_{i}, \gamma_{j}\right]$. We show $\nabla \gamma=0$ by checking:

$$
\begin{aligned}
& \gamma_{m ; m}=0, \\
& \gamma_{a ; m}=\partial_{r} f \cdot \gamma_{a}-\Gamma_{m a}{ }^{b} \gamma_{b}=0, \\
& \gamma_{m ; a}=-\Gamma_{a m}{ }^{b} \gamma_{b}+\left[\omega_{a}, \gamma_{m}\right]=-\partial_{r} f \cdot \gamma_{a}+\frac{1}{2} \partial_{r} f\left[\gamma_{m} \gamma_{a}, \gamma_{m}\right]=0, \\
& \gamma_{a ; b}=-\Gamma_{b a}{ }^{m} \gamma_{m}+\left[\omega_{b}, \gamma_{a}\right]=\partial_{r} f \cdot e^{2 f} \gamma_{m} \delta_{a b}+\frac{1}{2} \partial_{r} f\left[\gamma_{m} \gamma_{b}, \gamma_{a}\right]=0 .
\end{aligned}
$$

Consequently

$$
\begin{aligned}
& \psi_{P}=-\gamma^{a} \omega_{a}=-\frac{1}{2} \partial_{r} f \gamma^{a} \gamma_{m} \gamma_{a}=-\frac{1}{2}(m-1) \partial_{r} f \gamma_{m}, \\
& \psi_{A}=\gamma_{m} \gamma^{a} \omega_{a}+\delta_{2} \gamma_{0}=-\frac{1}{2}(m-1) \partial_{r} f+\delta_{2} \gamma_{0} .
\end{aligned}
$$

Thus $\gamma_{m} \psi_{P}-\psi_{P} \gamma_{m}=0$ and $\psi_{A}+\widetilde{\psi_{A^{\#}}}=L_{a a} \mathrm{Id}+2 \delta_{2} \gamma_{0}$. Furthermore as $\phi$ and $\rho$ are independent of $\theta, A+\widetilde{A^{\#}}=2 \delta_{2} \gamma_{0}$. Thus Equation (3.b) yields $c_{3}+c_{5}=0$ so, by Assertion (1), $c_{3}=-c_{5}=\frac{1}{2}$. This completes the proof of Lemma 3.3 and thereby the proof of Theorem 1.2

\section{ACKNOWLEDGMENTS}

Research of P. Gilkey partially supported by the Max Planck Institute for the Mathematical Sciences (Leipzig). Research of K. Kirsten partially supported by the Max Planck Institute for the Mathematical Sciences (Germany) and the Baylor University Summer Sabbatical Program. Research of JH. Park supported by R042000-00002 from the Korea Science and Engineering Foundation.

\section{REFERENCES}

[1] M.F. Atiyah, V.K. Patodi, and I.M. Singer. Spectral asymmetry and Riemannian geometry. Math. Proc. Camb. Phil. Soc. I, 77:43-69 1975; II 78:405-432, 1975; III, 79:71-99, 1976.

[2] M. van den Berg, S. Desjardins, and P.B. Gilkey. Functorality and heat content asymptotics for operators of Laplace type. Topological Methods in Nonlinear Analysis, 2:147-162, 1993.

[3] M. van den Berg and P.B. Gilkey. Heat content asymptotics of a Riemannian manifold with boundary. J. Funct. Anal., 120:48-71, 1994.

[4] S. Desjardins and P.B. Gilkey. Heat content asymptotics for operators of Laplace type with Neumann boundary conditions. Math. Z., 215:251-268, 1994.

[5] J.S. Dowker, P.B. Gilkey, and K. Kirsten. Heat asymptotics with spectral boundary conditions. Contemporary Math., 242:107-124, 1999. hep-th/0004020

[6] P. Gilkey. On the index of geometrical operators on Riemannian manifolds with boundary. Adv. in Math., 102:129-183, 1993.

[7] P.B. Gilkey and K. Kirsten. Heat asymptotics with spectral boundary conditions II. Proc. Roy. Soc. Edinb., 133A: 333-361, 2003. math-ph/0007015

[8] P.B. Gilkey, K. Kirsten, and JH. Park. Heat content asymptotics for oblique boundary conditions. Lett. Math. Phys., 59:269-276, 2002.

[9] P.B. Gilkey, K. Kirsten, and JH. Park. Heat content asymptotics for spectral boundary conditions. Trends in Mathematics, 5: 49-58, 2002. math-ph/0209012

[10] G. Grubb. Heat operator trace expansions and index for general Atiyah-Patodi-Singer boundary problems. Commun. Part. Diff. Equat., 17:2031-2077, 1992.

[11] G. Grubb. Trace expansions for pseudodifferential boundary problems for Dirac operators and more general systems. Ark. Math., 37:45-86, 1999.

[12] G. Grubb and R.T. Seeley. Asymptotic expansions for the Atiyah-Patodi-Singer operator. C.R. Acad. Sci., Paris, Ser. I, 317:1123-1126, 1993.

[13] G. Grubb and R.T. Seeley. Weakly parametric pseudodifferential-operators and AtiyahPatodi-Singer boundary-problems. Invent. Math., 121:481-529, 1995.

[14] G. Grubb and R.T. Seeley. Zeta and eta functions for Atiyah-Patodi-Singer operators. J. Geom. Anal., 6:31-77, 1996. 
[15] D.M. McAvity. Surface energy from heat content asymptotics. J. Phys. A: Math. Gen., 26:823-830, 1993.

[16] P. McDonald and R. Meyers. Dirichlet spectrum and heat content. J. Funct. Anal., 200: 150-159, (2003).

[17] A. Savo. Uniform estimates and the whole asymptotic series of the heat content on manifolds. Geometriae Dedicata, 73:181-214, 1998.

[18] A. Savo. On the asymptotic series of the heat content. Global differential geometry: the mathematical legacy of Alfred Gray. Proceedings of the international congress on differential geometry held in memory of Professor Alfred Gray, Bilbao, Spain, September 18-23, 2000, Ed. M. Ferández, Marisa et al., Contemp. Math., 288:428-432, (2001).

Mathematics Department, University of Oregon, Eugene Or 97403 USA

E-mail address: gilkey@darkwing.uoregon.edu

Department of Mathematics, Baylor University, Waco, TX 76798, USA

E-mail address: Klaus_Kirsten@Baylor.edu

Department of Computer and Applied Mathematics, Honam University, Gwangue 506714 Korea

E-mail address: jhpark@honam.ac.kr 